\begin{tabular}{|c|l|}
\hline Title & Large thermoelectric voltage in point contacts of Ni ferromagnetic metals \\
\hline Author(s) & Kondo, Kenji; Kaiju, Hideo; Ishibashi, A kira \\
\hline Citation & $\begin{array}{l}\text { MRS Proceedings, 1314, mrsf10-1314/I08-36 } \\
\text { https://doi.org/10.1557/opl.2011.267 }\end{array}$ \\
\hline Issue Date & 2011-03-08 \\
\hline Doc URL & http://hdl.handle.net/2115/57072 \\
\hline Rights & ○ 2011 Materials Research Society \\
\hline Type & article \\
\hline File Information & Large Thermoelectric V oltage in Point Contacts of Ni Ferromagnetic Metals.pdf \\
\hline
\end{tabular}

Instructions for use 


\title{
Large Thermoelectric Voltage in Point Contacts of Ni Ferromagnetic Metals
}

Kenji Kondo ${ }^{1}$, Hideo Kaiju ${ }^{1,2}$, and Akira Ishibashi ${ }^{1}$

${ }^{1}$ Laboratory of Quantum Electronics, Research Institute for Electronic Science, Hokkaido University, Sapporo 001-0020, Japan.

${ }_{2}^{2}$ PRESTO, Japan Science and Technology Agency, Saitama 332-0012, Japan.

\begin{abstract}
Recently, we have proposed a spin quantum cross structure (SQCS) device toward the realization of novel spintronics devices. In this paper, we have investigated thermoelectric effects in point contacts (PCs) of $\mathrm{Ni}$ ferromagnetic metals using SQCS devices, theoretically and experimentally. The calculated results show that the thermoelectric voltage $V_{q}$ changes from 0.48 $\mathrm{mV}$ to $2.12 \mathrm{mV}$ with the temperature difference of PCs increasing from $10 \mathrm{~K}$ to $50 \mathrm{~K}$. Also, the magnitude of the theoretical thermoelectric voltage agrees very well with that of the experimental result. PCs of SQCS devices with Ni electrodes can serve as spin dependent thermobatteries.
\end{abstract}

\section{INTRODUCTION}

Many researchers have paid much attention to point contacts (PCs) of ferromagnetic metals since conductance quantization in units of $e^{2} / h$ was discovered at PCs of Ni ferromagnetic metals [1]. Also, some researchers report that PCs of $\mathrm{Ni}$ ferromagnetic metals show large magnetoresistance in the ballistic regime [2]. However, thermal characteristics in PCs of ferromagnetic metals have almost not been studied even though those in PCs of normal metals have been extensively studied. In this paper, we have theoretically investigated thermoelectric effects in PCs of Ni ferromagnetic metals using spin quantum cross structure (SQCS) devices, and we have verified the thermoelectric effects in PCs of Ni ferromagnetic metals experimentally. The SQCS device consists of two ferromagnetic metal thin films with their edges crossing, and sandwiches a few molecules and atoms [3-6]. The junction area made of two edges can be scaled down to nanometer size due to the good resolution in making films by the metal-deposition rate, ranging from $0.01 \mathrm{~nm} / \mathrm{s}$ to $1 \mathrm{~nm} / \mathrm{s}$. We have already realized the SQCS device with $\mathrm{Ni}$ ferromagnetic electrodes [6].

\section{THEORY AND EXPERIMENTAL RESULTS}

The models of SQCS devices with two-dimensional (2D) electrodes are shown in figure 1(a). We have analyzed the spin dependent thermoelectric characteristics within the framework of the Anderson Hamiltonian. We study the current-voltage (I-V) characteristics of SQCS devices with a molecule sandwiched between two $\mathrm{Ni}$ magnetic electrodes. The molecule is assumed to have two energy levels. We also assume that the molecule does not flip the spin of an electron passing through the energy level. The energy diagram for the SQCS device model is also shown in figure 1(b). Notice that this model can be applied to SQCS devices without any molecules sandwiched between the two Ni magnetic electrodes. In the case that there are no sandwiched molecules, both electrodes are in contact with each other directly and the conductance channels (energy levels) are formed in the gap between the electrodes. The energy 
levels formed in the gap between the electrodes can be regarded as energy levels which the virtual molecule has, as if the virtual molecule was in the gap. This concept has been verified to work very well in a previous paper [6].

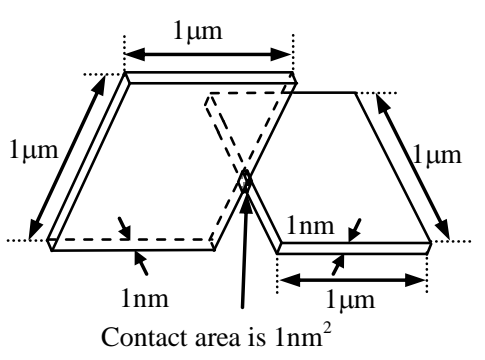

(a) SQCS model with 2D electrodes

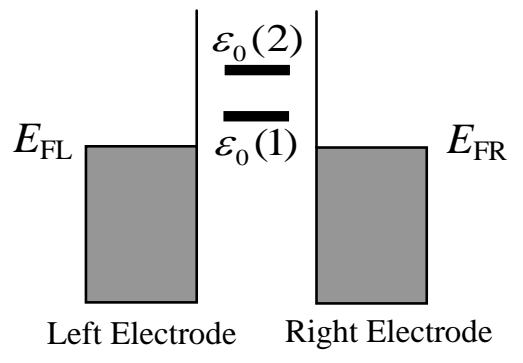

(b) Energy diagram for SQCS devices

Figure 1. Schematic illustration of (a) SQCS device models with two-dimensional (2D) electrodes and (b) the energy diagram for SQCS devices.

The Anderson Hamiltonian is described as follows:

$$
\begin{aligned}
& H=H_{\text {Electrodes }}+H_{\text {mole }}+H_{\mathrm{t}}, \\
& H_{\text {Electrodes }}=\sum_{\alpha=L, R} \sum_{\boldsymbol{k}, \sigma} \varepsilon_{\boldsymbol{k} \sigma} c_{\alpha, \boldsymbol{k} \sigma}^{+} c_{\alpha, \boldsymbol{k} \sigma}, \\
& H_{\text {mole }}=\sum_{i, \sigma} \varepsilon_{0}(i) a_{i, \sigma}^{+} a_{i, \sigma}, \\
& H_{\mathrm{t}}=\sum_{\alpha=L, R} \sum_{k, \sigma} \sum_{i, \sigma}\left(V_{\alpha}^{\sigma} c_{\alpha, \boldsymbol{k} \sigma}^{+} a_{i, \sigma}+\text { H.c. }\right) .
\end{aligned}
$$

$H_{\text {Electrodes }}$ is the Hamiltonian of both Ni electrodes, $\varepsilon_{\boldsymbol{k} \sigma}=\frac{\hbar^{2} \boldsymbol{k}^{2}}{2 m}, m$ is the free electron mass, and $\hbar$ is Planck's constant $h$ divided by $2 \pi$. The band of itinerant d-like electrons of Ni is assumed to be parabolic with the free electron mass [7]. The wave vector $\boldsymbol{k}$ is a 2D vector in 2D electrodes. $c_{\alpha, k \sigma}^{+}$and $c_{\alpha, k \sigma}$ are creation and annihilation operators for electrons of wave vector $\boldsymbol{k}$ and spin index $\sigma$ in $\alpha$ electrode. $\alpha$ indicates the left or right electrode. The creation and annihilation operators obey the standard fermion anticommunication rules. $H_{\text {mole }}$ is the Hamiltonian of a molecule sandwiched between both the electrodes, and $\varepsilon_{0}(i)$ represents the $i$-th energy level of eigenstates of the molecule as shown in figure 1(b). In this paper, we consider the case that both electrodes of SQCS devices are in contact with each other directly. Then, the two energy levels were set to be $\varepsilon_{0}(1)=10 \mathrm{meV}, \varepsilon_{0}(2)=20 \mathrm{meV}$, estimated from Fermi levels $E_{\mathrm{FL}}, E_{\mathrm{FR}}$ of each electrode, respectively. These energy levels are assumed to be quantized levels formed in a narrow gap between the electrodes. The Fermi levels $E_{\mathrm{FL}}$ and $E_{\mathrm{FR}}$ of each electrode were assumed to be equal and we used the value of $9.071 \mathrm{eV}$ for the Ni Fermi level [8]. The value of $260 \mathrm{meV}$ was used for the exchange splitting of $\mathrm{Ni}$ [9]. $a_{i, \sigma}^{+}$and $a_{i, \sigma}$ are creation and annihilation operators for electrons of spin index $\sigma$ in the $i$-th energy level. These operators also obey the 
standard fermion anticommunication rules. $H_{\mathrm{t}}$ is the transfer Hamiltonian between the sandwiched molecule and each electrode, $V_{\alpha}^{\sigma}$ is the spin-dependent transfer matrix between the molecule and electrons of spin index $\sigma$ in $\alpha$ electrode. This value determines the coupling strength between each electrode and the molecule. Considering $H_{\mathrm{t}}$ as a perturbation, we have investigated the $I-V$ characteristics from right to left electrode. After tedious manipulation, the current $I_{\uparrow \uparrow}\left(I_{\uparrow \downarrow}\right)$ from right to left electrode is as follows:

$$
\begin{aligned}
I_{\uparrow \uparrow}=\frac{e}{h} \int_{E_{\mathrm{FR}}}^{E_{\mathrm{FR}}+e V} d \varepsilon \sum_{i}\left(\frac{4 \Gamma_{\mathrm{L}}^{+}(\varepsilon) \Gamma_{\mathrm{R}}^{+}(\varepsilon)}{\left[\varepsilon-\varepsilon_{0}(i)\right]^{2}+\left[\Gamma^{++}(\varepsilon)\right]^{2}}+\frac{4 \Gamma_{\mathrm{L}}^{-}(\varepsilon) \Gamma_{\mathrm{R}}^{-}(\varepsilon)}{\left[\varepsilon-\varepsilon_{0}(i)\right]^{2}+\left[\Gamma^{--}(\varepsilon)\right]^{2}}\right) \\
\times\left[f\left(\varepsilon-e V-E_{\mathrm{FR}}, T_{\mathrm{L}}\right)-f\left(\varepsilon-E_{\mathrm{FR}}, T_{\mathrm{R}}\right)\right], \\
I_{\uparrow \downarrow}=\frac{e}{h} \int_{E_{\mathrm{FR}}}^{E_{\mathrm{FR}}+e V} d \varepsilon \sum_{i}\left(\frac{4 \Gamma_{\mathrm{L}}^{+}(\varepsilon) \Gamma_{\mathrm{R}}^{-}(\varepsilon)}{\left[\varepsilon-\varepsilon_{0}(i)\right]^{2}+\left[\Gamma^{+-}(\varepsilon)\right]^{2}}+\frac{4 \Gamma_{\mathrm{L}}^{-}(\varepsilon) \Gamma_{\mathrm{R}}^{+}(\varepsilon)}{\left[\varepsilon-\varepsilon_{0}(i)\right]^{2}+\left[\Gamma^{-+}(\varepsilon)\right]^{2}}\right) \\
\times\left[f\left(\varepsilon-e V-E_{\mathrm{FR}}, T_{\mathrm{L}}\right)-f\left(\varepsilon-E_{\mathrm{FR}}, T_{\mathrm{R}}\right)\right],
\end{aligned}
$$

where $e$ is the elementary charge, $I_{\uparrow \uparrow}\left(I_{\uparrow \downarrow}\right)$ is the current flowing from right to left electrode when two Ni magnetic electrodes have parallel (antiparallel) magnetic moments, $T_{\mathrm{L}(\mathrm{R})}$ is the temperature of left (right) electrode, $\Gamma^{++}(\varepsilon)=\Gamma_{\mathrm{L}}^{+}(\varepsilon)+\Gamma_{\mathrm{R}}^{+}(\varepsilon), \Gamma^{--}(\varepsilon)=\Gamma_{\mathrm{L}}^{-}(\varepsilon)+\Gamma_{\mathrm{R}}^{-}(\varepsilon)$, $\Gamma^{+-}(\varepsilon)=\Gamma_{\mathrm{L}}^{+}(\varepsilon)+\Gamma_{\mathrm{R}}^{-}(\varepsilon), \Gamma^{-+}(\varepsilon)=\Gamma_{\mathrm{L}}^{-}(\varepsilon)+\Gamma_{\mathrm{R}}^{+}(\varepsilon)$, and $f(\varepsilon, T)=1 /\left(\exp \left(\varepsilon /\left(k_{\mathrm{B}} T\right)\right)+1\right)$ is the Fermi-Dirac distribution function, where $T$ is the temperature and $k_{\mathrm{B}}$ is Boltzmann's constant. Since both the electrodes are the same $\mathrm{Ni}$, the equations $\Gamma^{+-}(\varepsilon)=\Gamma^{-+}(\varepsilon), \Gamma_{\mathrm{L}}^{+}(\varepsilon)=\Gamma_{\mathrm{R}}^{+}(\varepsilon)$, $\Gamma_{\mathrm{L}}^{-}(\varepsilon)=\Gamma_{\mathrm{R}}^{-}(\varepsilon)$ hold from the symmetry. The superscript $+(-)$ designates the coupling between the density of states of the majority (minority) spin electrons and the molecule. Then, $\Gamma_{\mathrm{L}}^{+}(\varepsilon), \Gamma_{\mathrm{R}}^{+}(\varepsilon)\left(\Gamma_{\mathrm{L}}^{-}(\varepsilon), \Gamma_{\mathrm{R}}^{-}(\varepsilon)\right)$ are coupling strengths between the molecule and the majority (minority) spin electrons of left and right electrodes, respectively. Changing the temperature difference $\Delta T_{\mathrm{PC}}$ of PCs, we have calculated the thermoelectric transport characteristics of SQCS devices at ambient temperature $T_{\mathrm{a}}=300 \mathrm{~K}$. In this case, we used the coupling strengths $\Gamma_{\mathrm{L}(\mathrm{R})}^{+}(\varepsilon)$ of $47.2 \mu \mathrm{eV}$ and $\Gamma_{\mathrm{L}(\mathbb{R})}^{-}(\varepsilon)$ of $0.47 \mu \mathrm{eV}$. Figures 2(a) and 2(b) show the calculated thermoelectric characteristics when two $\mathrm{Ni}$ electrodes have parallel magnetic moments, and when two $\mathrm{Ni}$ electrodes have antiparallel magnetic moments, respectively. From figures 2(a) and 2(b), we can notice that the thermoelectric voltage $V_{q}$ changes from $0.48 \mathrm{mV}$ to $2.12 \mathrm{mV}$ with the temperature difference $\Delta T_{\mathrm{PC}}$ of PCs increasing from $10 \mathrm{~K}$ to $50 \mathrm{~K}$. Also, the magnitude of the thermoelectric voltage $V_{q}$ is independent of spin directions and the thermopower $S$ is $42.4 \mu \mathrm{V} / \mathrm{K}$ for both spin directions. On the other hand, the maximum power $P_{\max }$ extracted from PCs of SQCS devices is dependent on spin directions. The maximum power $P_{\max }$ can be computed from the $I-V$ curve under the fourth quadrant. $P_{\max }$ 's are $5.14 \mathrm{pW}, 0.105 \mathrm{pW}$ at $\Delta T_{\mathrm{PC}}=50 \mathrm{~K}$ for SQCS devices when two Ni electrodes have parallel and antiparallel magnetic moments, respectively. Therefore, we consider that PCs of SQCS devices can serve as spin dependent thermobatteries. 

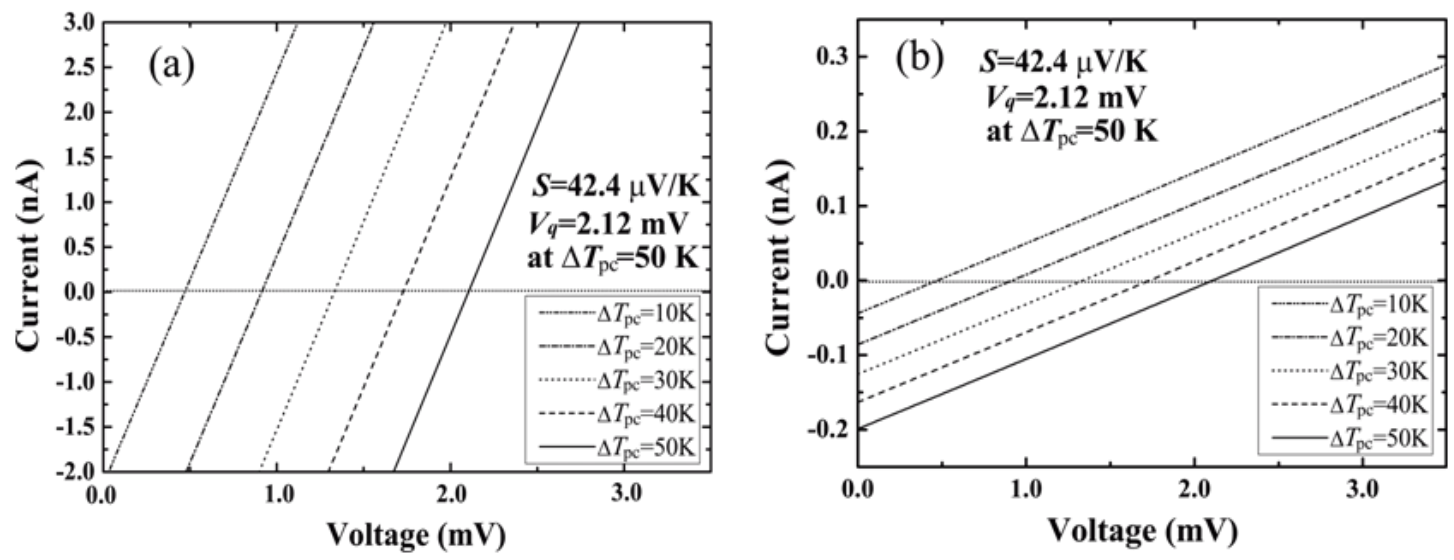

Figure 2. Thermoelectric transport characteristics of SQCS devices when two Ni electrodes have (a) parallel magnetic moments and (b) antiparallel magnetic moments.

We have realized an SQCS device with Ni thin film electrodes. Ni thin films have been thermally evaporated on polyethylene naphthalate (PEN) substrates $(2 \mathrm{~mm}$ width, $10 \mathrm{~mm}$ length and $100 \mu \mathrm{m}$ thickness) in a high vacuum chamber at a base pressure of $10^{-8}$ Torr. Fabricated Ni/PEN films have been sandwiched between two polymethyl methacrylate (PMMA) resins and the edge of PMMA/Ni/PEN/PMMA has been polished by chemical mechanical polishing (CMP) methods. Two sets of polished PMMA/Ni/PEN/PMMA have been prepared and attached together with their edges crossing. Figure 3(a) shows the schematic fabrication method of Ni SQCS devices. The evaporated Ni thin film is $2 \mathrm{~mm}$ wide, $10 \mathrm{~mm}$ long, and $17 \mathrm{~nm}$ thick. Figure 3(b) shows the experimental results of thermoelectric effects at PCs in Ni SQCS devices. The SQCS device was at room temperature $294.5 \mathrm{~K}$ at first. When the ambient temperature changes to $295.5 \mathrm{~K}$, we notice that a large thermoelectric voltage $V_{q}$ has emerged as shown in figure 3(b).
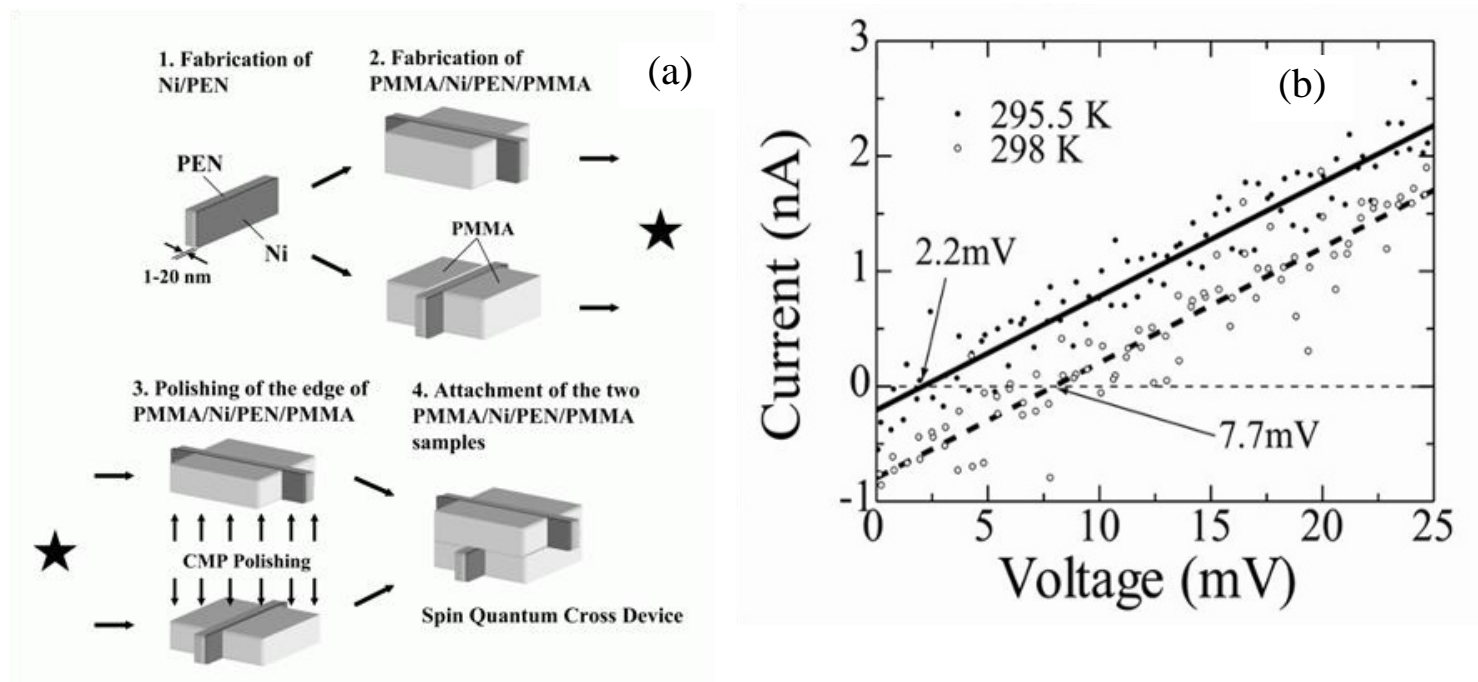

Figure 3. (a) Schematic fabrication method of Ni SQCS devices and (b) experimental results of thermoelectric effect of Ni SQCS devices.

Also, $V_{q}$ changes from $2.2 \mathrm{mV}$ to $7.7 \mathrm{mV}$ when the ambient temperature changes from $295.5 \mathrm{~K}$ to $298 \mathrm{~K}$. According to the above theory, the temperature at PCs of SQCS devices must be about 
$50 \mathrm{~K}$ in order to explain the result at $295.5 \mathrm{~K}$. You may think that a temperature difference of 50 $\mathrm{K}$ is large. However, the difference can easily occur in the case that the thermal conductivities of the two electrodes differ from each other by one order of magnitude and that the process of heat transfer is transient. In this experiment, the thermal conductivity of one electrode is $\kappa_{\mathrm{L}}=1.7 \times 10^{-2}$ $\mathrm{W} / \mathrm{cmK}$ and that of the other electrode is $\kappa_{\mathrm{R}}=1.7 \times 10^{-3} \mathrm{~W} / \mathrm{cmK}$. The change in ambient temperature causes a thermal current between the two electrodes so that the temperature of the two electrodes approaches the ambient temperature. This process is much longer than expected, and it takes more than 15 minutes to reach equilibrium. The process can be regarded as transient during the $I-V$ measurement. Figure 4(a) shows the calculated temperature difference between the Ni electrodes with different thermal conductivities by solving the one-dimensional thermal diffusion equation using the finite element method, assuming that only the edges of the electrodes are exposed to the ambient temperature. This approximation is very accurate because the other parts of the electrodes are covered with PMMA which is a heat insulating material. The coordinate systems for finite element method are shown in figure 4(b).

(a)

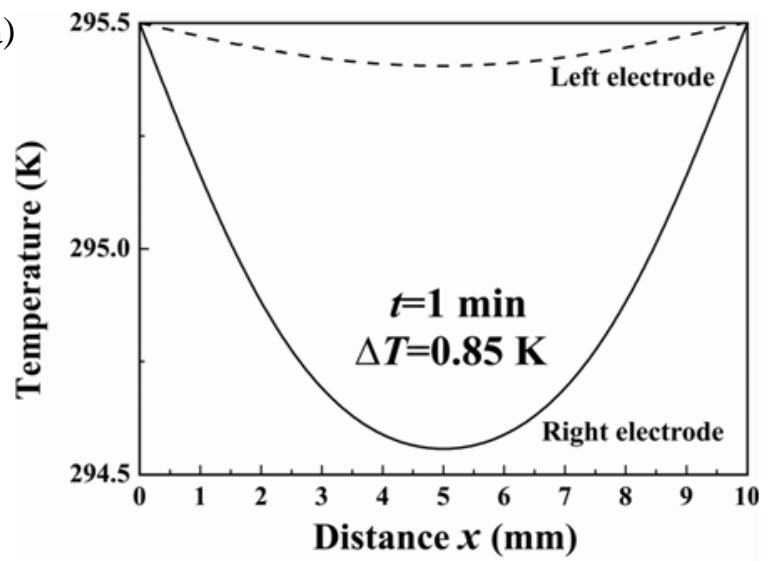

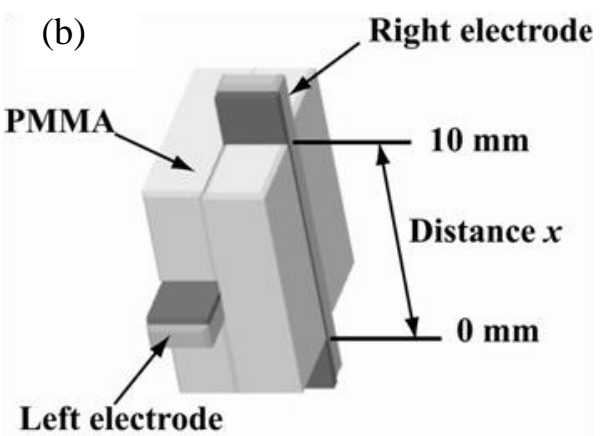

Spin Quantum Cross Device

Figure 4. (a) Temperature difference between $\mathrm{Ni}$ electrodes with different thermal conductivities. (b) The coordinate systems for finite element method.

When the ambient temperature changes from $294.5 \mathrm{~K}$ to $295.5 \mathrm{~K}$, the temperature of the left electrode with large thermal conductivity is almost $295.5 \mathrm{~K}$ after 1 minute. On the other hand, the temperature of the middle point (cross point) of the right electrode with small thermal conductivity remains almost unchanged even after 1 minute. Therefore, the temperature difference $\Delta T$ of $0.85 \mathrm{~K}$ arises at close proximity of the PC. As a result, a thermal flux is generated and flows into the PC of the SQCS device from the electrode. However, the thermal flux is disturbed at the PC of the device because the thermal conductance of the PC is as small as $6.58 \times 10^{-11} \mathrm{~W} / \mathrm{K}$, which is estimated from the following equation:

$$
\kappa_{\mathrm{PC}}=L_{0} G T_{a}\left(1-S^{2} / L_{0}\right),
$$

where $L_{0}$ is the Lorentz number, and $G$ is the conductance [10]. Therefore, a large temperature difference $\Delta T_{\mathrm{PC}}$ at $\mathrm{PC}$ occurs in order to allow the same thermal flow through the $\mathrm{PC}$ as that through the electrodes, as shown in the following equation:

$$
-\kappa_{\mathrm{PC}} \Delta T_{\mathrm{PC}}=J_{\mathrm{t}} \approx-\kappa_{\mathrm{L}} \Delta d \Delta T .
$$

$J_{\mathrm{t}}$ is the thermal flow estimated at close proximity to the PC. Then, $\kappa_{\mathrm{L}} \Delta d$ is the thermal conductance of the electrodes at close proximity of the PC, and we can ignore the thermal 
conductance of the right electrode because its thermal conductance is smaller than that of the left electrode by one order of magnitude. If we assume that the gap $\Delta d$ between the two $\mathrm{Ni}$ electrodes is $2.5 \mathrm{~nm}$, we can estimate $\Delta T_{\mathrm{PC}}$ to be $54.90 \mathrm{~K}$ at the PC. According to the above theory, the thermopower $S$ is $42.4 \mu \mathrm{V} / \mathrm{K}$. Then, $V_{q}$ is $2.33 \mathrm{mV}$, which is in very good agreement with the experimental value of $2.2 \mathrm{mV}$. Using similar analysis, when the ambient temperature changes from $294.5 \mathrm{~K}$ to $298 \mathrm{~K}$, the temperature difference $\Delta T$ of $2.97 \mathrm{~K}$ arises at close proximity of the PC. Therefore, we can estimate $\Delta T_{\mathrm{PC}}$ to be $191.83 \mathrm{~K}$ at the PC. In this case, $V_{q}=S \Delta T_{\mathrm{PC}}$ is $8.13 \mathrm{mV}$, which agrees very well with the experimental value of $7.7 \mathrm{mV}$. These very good agreements mean that the developed theory is very effective.

\section{CONCLUSIONS}

In this paper, we have investigated thermoelectric effects in point contacts (PCs) of $\mathrm{Ni}$ ferromagnetic metals using SQCS devices, which we have proposed as novel spintronics devices, theoretically and experimentally. The calculated results show that the thermoelectric voltage $V_{q}$ changes from $0.48 \mathrm{mV}$ to $2.12 \mathrm{mV}$ with the temperature difference of PCs increasing from $10 \mathrm{~K}$ to $50 \mathrm{~K}$. Also, the magnitude of the thermoelectric voltage $V_{q}$ is independent of spin directions and the thermopower $S$ is $42.4 \mu \mathrm{V} / \mathrm{K}$ for both spin directions. On the other hand, the maximum power $P_{\max }$ extracted from PCs of SQCS devices is dependent on spin directions. Then, PCs of SQCS devices with Ni electrodes can serve as spin dependent thermobatteries. Also, we have measured thermoelectric effects in PCs of SQCS devices experimentally. The magnitude of the experimental thermoelectric voltage agrees very well with that of the theoretical result.

\section{ACKNOWLEDGMENTS}

This research has been partially supported by Special Education and Research Expenses from Post-Silicon Materials and Devices Research Alliance, also by a Grant-in-Aid for Scientific Research from Japan Society for the Promotion of Science, and Research for Promoting Technological Seeds from JST.

\section{REFERENCES}

1. H. Oshima and K. Miyano, Appl. Phys. Lett. 73, 2203 (1998).

2. N. Garcia, M. Munoz, and Y.-W. Zhao, Phys. Rev. Lett. 82, 2923 (1999).

3. K. Kondo and A. Ishibashi, Jpn. J. Appl. Phys. 45, 9137 (2006).

4. H. Kaiju, K. Kondo, and A. Ishibashi, Mater.Res.Soc.Symp.Proc. 961, O5.5.1 (2007).

5. K. Kondo, H. Kaiju, and A. Ishibashi, Mater.Res.Soc.Symp.Proc. 1067, B03-01 (2008).

6. K. Kondo, H. Kaiju, and A. Ishibashi, J. Appl. Phys. 105, 07D522 (2009).

7. M. B. Stearns, J. Magn. Magn. Mater. 5, 167 (1977).

8. C. S. Wang and J. Callaway, Phys. Rev. B 15, 298 (1977).

9. W. Eberhardt and E. W. Plummer, Phys. Rev. B 21, 3245 (1980).

10. H. van Houten, L. W. Molenkamp, C. W. J. Beenakker, and C. T. Foxon, Semicond. Sci. Technol. 7, B215 (1992). 